\title{
A comparison of three methods of orienting cervical punch biopsies
}

\author{
Mark K Heatley
}

\begin{abstract}
Aims-To compare methods of orienting cervical punch biopsies, since well oriented biopsies are needed to optimise the diagnosis and grading of cervical intraepithelial neoplasia.

Methods-The orientation, the presence and preservation of the squamocolumnar junction, and the presence or absence of the surface layers of the squamous epithelium were compared in 345 cervical biopsies that had either been attached to paper $(n=112)$, floated directly into $10 \%$ formalin $(n=107)$, or floated into a solution of $10 \%$ formalin including $0.05 \%$ eosin $(n=126)$ in the colposcopy clinic.

Results-When the specimens were mounted on filter paper before fixation, they were more likely to be optimally oriented, to have a preserved squamocolumnar junction, and to have intact surface epithelium than specimens that were handled using the other methods, even when the specimens floated off the paper in transit.

Conclusions-Cervical biopsy specimens should be mounted on paper before fixation and submission to the laboratory. (F Clin Pathol 1999;52:149-150)
\end{abstract}

Keywords: cervix uteri; biopsy; quality control; laboratory diagnosis

It is necessary to have well oriented histological sections for an accurate assessment of the presence and grade of cervical intraepithelial neoplasia (CIN) in cervical punch biopsies. ${ }^{12}$

In some other branches of anatomical pathology, small biopsies are simply removed from the biopsy forceps and placed in fixative, to avoid inducing traumatic artefact. ${ }^{3}$ Gastrointestinal mucosal biopsies, however, have an attached muscularis mucosae and so tend to curl up with their luminal mucosa outwards so that they can be readily embedded. ${ }^{3}$ The cervix lacks a muscularis and is thus more difficult to orient. In the past various methods have been suggested to achieve this, including orienting the freshly taken biopsy on filter paper $^{1}$ or cucumber, ${ }^{4}$ and the use of insoluble glues. ${ }^{5}$

To determine whether the process of prefixation orientation of the specimen improves the quality of the ultimate histological section, an assessment was made of the orientation of a series of cervical biopsies that had been mounted on filter paper, comparing it with the orientation of biopsies that had simply been shaken off the biopsy forceps into a pot of standard fixative, and with biopsies that were shaken into a $0.05 \%$ solution of eosin in $10 \%$ formalin. The aim of including eosin in the fixative was to improve the differentiation between epithelium and stroma, thereby facilitating orientation when the biopsy was embedded before sectioning.

\section{Methods}

Routine cervical punch biopsies taken in the colposcopy clinic $(n=112)$ were eased out of the biopsy forceps cup by use of a teasing pin and mounted on filter paper when fresh, before fixation. The biopsy, attached stromal surface down to the filter paper, was allowed to adhere for a few moments and dropped into $10 \%$ unbuffered formalin. A second group ( $n=107)$ consisted of a series of cases reported over a comparable time period one year before the system of mounting cervical biopsies was introduced. These had been shaken from the biopsy forceps into fixative with no attempt to orient the specimen in the clinic. A final group $(n=126)$ consisted of a series of biopsies that were shaken into fixative which consisted of a $0.05 \%$ solution of eosin in formalin.

Upon receipt of the biopsy in the laboratory, an experienced medical laboratory scientific officer measured its dimensions and transferred it, still attached to the filter paper, to a processing cassette. The biopsy was embedded in paraffin wax and three $5 \mu \mathrm{m}$ sections were cut at different levels in the block, mounted on a single slide, and stained with haematoxylin and eosin.

The slides were reviewed by a single pathologist who assessed the following features.

(1) The orientation of the biopsy was classified in to one of four categories-perfect orientation; orientation up to $30^{\circ}$ from the true axis; orientation between $30^{\circ}$ and $60^{\circ}$ from the true axis; and orientation more than $60^{\circ}$ from the true axis, a category that included specimens embedded en face.

(2) The presence or absence of the squamocolumnar junction and if present, whether it was disrupted or well preserved.

(3) The presence or absence of trauma to the superficial layers of the ectocervical squamous epithelium.

STATISTICAL ANALYSIS

Statistical analyses were performed using the $\chi^{2}$ test. A p value of less than 0.05 was considered significant.

\section{Results}

The quality of orientation, ability to identify the squamocolumnar junction, and the presence or absence of an intact squamous 
Table 1 Effects of three techniques for fixing cervical biopsies in the colposcopy clinic on orientation as classified using a four point scale, presence or absence of the squamocolumnar junction, and presence or absence of trauma to the surface layers of squamous epithelium

\begin{tabular}{lllll}
\hline & & $\begin{array}{l}\text { Floated in } 10 \% \\
\text { formalin }\end{array}$ & Eosin in formalin & \\
\hline Orientation & $58(52 \%)$ & $31(29 \%)$ & $22(17 \%)$ & \\
$\quad \begin{array}{l}\text { Perfect } \\
\text { Up to } 30^{\circ}\end{array}$ & $33(30 \%)$ & $41(39 \%)$ & $54(43 \%)$ & \\
$30^{\circ}$ to $60^{\circ}$ & $13(11 \%)$ & $26(24 \%)$ & $30(24 \%)$ & \\
$\quad$ Over $60^{\circ}$ & $8(7 \%)$ & $9(8 \%)$ & $20(16 \%)$ & a,c,d \\
Squamocolumnar junction & & & & \\
$\quad$ Identified & $36(32 \%)$ & $18(17 \%)$ & $13(10 \%)$ & \\
$\quad$ Not present & $50(45 \%)$ & $49(46 \%)$ & $58(46 \%)$ & b,c,d \\
$\quad$ Traumatised & $26(23 \%)$ & $40(37 \%)$ & $55(44 \%)$ & \\
Surface epithelium & $18(16 \%)$ & $30(29 \%)$ & $54(43 \%)$ & b,c,e \\
$\quad$ Missing & $94(84 \%)$ & $76(71 \%)$ & $72(57 \%)$ & \\
$\quad$ Intact & 112 & 107 & 126 & \\
Total (n) & & & & \\
\hline
\end{tabular}

Specimens were either allowed to adhere to filter paper before being placed in fixative, or placed directly into fixative, or placed in fixative containing dye which it was hoped would assist in distinguishing between stroma and epithelium. All statistical analyses were performed using the $\chi^{2}$ test.

Attached to paper $v$ floated in formalin, a: $0.001<$ p $<0.01$; b: $0.01<$ p $<0.05$.

Attached to paper $v$ eosin in formalin, $\mathrm{c}: \mathrm{p}<0.0001$

Floated in formalin $v$ eosin in formalin, $\mathrm{d}$ : $\mathrm{p}>0.05$; e: $0.01<\mathrm{p}<0.05$.

epithelium for each of the three study groups are shown in table 1 .

The best orientation was achieved when the specimens were mounted on paper before fixation. Sixty two biopsies remained attached and 50 detached from the filter paper before receipt in the laboratory. The quality of orientation was similar in the two groups (attached $v$ detached, $\mathrm{p}=0.54$ ). The squamocolumnar junction was less likely to be traumatised when the specimen had been mounted on paper. Equally, the superficial epithelium was most likely to remain intact if the specimen had been placed on paper.

\section{Discussion}

The study provides evidence to support the practice of mounting cervical biopsy specimens before submitting the biopsy to the laboratory. Better specimen orientation was achieved and an intact squamocolumnar junction was more likely to be identified when the specimen was mounted on paper than when the biopsy was placed straight into fixative. There was no evi- dence that orientation was affected when the biopsy floated off the filter paper. In this situation orientation was facilitated because the biopsy continued to have a flat base which permitted the stroma to be identified and thus assisted in orientation.

Adding eosin to the fixative solution did not improve orientation, possibly because there was no difference in the staining of epithelium and stroma. This resulted in greater difficulty in orienting these samples before embedding, with a subsequent high incidence of poorly oriented specimens.

It is tempting to attribute the variation with which the squamocolumnar junction was identified and the ability to recover an untraumatised epithelial surface to operator expertise in the colposcopy clinic. However, since there was no significant change in staffing of the clinic or the laboratory between each of the study periods it is more probable that this variation reflects the method by which tissue in each of the three study groups was handled. It has been demonstrated that shaking gastrointestinal specimens into fixative causes fragmentation of small biopsies, especially those originating from the duodenum. The loss of ectocervical epithelium from the surface of the cervix and disruption of the squamocolumnar junction may reflect this process at another anatomical site. $^{3}$

I am grateful to Mr C Heepy and Mrs B Jones, Department of I am grateful to Mr C Heepy and Mrs B Jones, Department of
Cellular Pathology, Taunton and Somerset NHS Trust for their help in performing this study.

1 Coleman DV, Evans DMD. Biopsy pathology and cytology of the cervix. London: Chapman and Hall, 1988:27-8.

2 Kuesel AC, Kroft T, Saunders JK, et al. A simple procedure for obtaining high-quality NMR spectra of semiquantitative value from small tissue specimens: cervical biopsies. Magn Reson Med 1992;27:349-55.

3 Day DW, Dixon MF. Biopsy pathology of the oesophagus and duodenum, 2nd ed. London: Chapman and Hall, 1995:1-3. 4 Swan RW, Davis HJ. The biopsy-cucumber unit. A method to improve tissue orientation. Obstet Gynecol 1970;36:8035.

5 Karnauchow PN. Small biopsy orientation. Arch Pathol Lab Med 1989;113:111. 